\title{
Lexis
}

Journal in English Lexicology

Book reviews | 2009

\section{Michel SIMON, Lexique et syntaxe - Lexicalisation des verbes complexes anglais}

Travaux du C.I.E.L., Université Paris 7 - Denis Diderot. 2005-2006, 191 pages

\section{Antoine Consigny}

\section{OpenEdition}

\section{Journals}

Édition électronique

URL : http://journals.openedition.org/lexis/1763

DOI : $10.4000 /$ lexis. 1763

ISSN : 1951-6215

\section{Éditeur}

Université Jean Moulin - Lyon 3

Référence électronique

Antoine Consigny, "Michel sımon, Lexique et syntaxe - Lexicalisation des verbes complexes anglais ", Lexis [En ligne], Recensions, mis en ligne le 17 août 2009, consulté le 24 septembre 2020. URL : http:// journals.openedition.org/lexis/1763; DOI : https://doi.org/10.4000/lexis.1763

Ce document a été généré automatiquement le 24 septembre 2020.

\section{(i)

Lexis is licensed under a Creative Commons Attribution-NonCommercial-NoDerivatives 4.0 International License. 


\section{Michel SIMON, Lexique et syntaxe - Lexicalisation des verbes complexes anglais}

Travaux du C.I.E.L., Université Paris 7 - Denis Diderot. 2005-2006, 191 pages

\section{Antoine Consigny}

\section{RÉFÉRENCE}

Michel Simon

Lexique et syntaxe - Lexicalisation des verbes complexes anglais. Travaux du C.I.E.L., Université Paris 7 - Denis Diderot. 2005-2006. ISBN : 290673127-7, Prix : $12 €, 191$ pages

1 Le livre compte 192 pages, dont 10 pages d'introduction par C.Cortès, 2 pages de bibliographie, 9 pages d'annexes, ainsi qu'une page de liste des sources et une autre pour la table des matières. Version remaniée d'une thèse de doctorat, c'est un véritable compte-rendu de recherche, qui s'adresse principalement à un public averti et au fait des concepts et de la terminologie utilisés en linguistique énonciative.

Le corps du texte est composé de 3 chapitres : dans le premier, qui est une introduction générale, M. Simon présente son sujet, le cadre théorique et la méthodologie utilisée pour l'étude. Le second est une étude des "phrasal verbs", selon la méthodologie définie précédemment, et dans le troisième, l'étude se tourne vers les verbes préfixés ou "compound verbs", en gardant la même méthodologie et en comparant les résultats avec la partie précédente.

Le chapitre premier commence naturellement par une définition des termes de "phrasal verb», de " prepositional verb» et de « compound verb». Les premiers sont une unité formée d'un verbe et d'une particule adverbiale d'origine directionnelle, placée à droite du verbe et graphiquement séparée de celui-ci ; les deuxièmes diffèrent des premiers en ceci que la "particule» n'est pas de même nature. Au lieu d'une 
particule adverbiale, c'est une préposition qui suit le verbe. Syntaxiquement, la différence a plusieurs conséquences, qui sont autant de critères simples et efficaces de distinction entre "phrasal verb» et «prepositional verb». D'abord, la préposition introduit un syntagme prépositionnel, formé de la préposition suivie d'un syntagme nominal. Ce qui se traduit par un ordre des mots plus figé pour le verbe prépositionnel : il est quasiment impossible de séparer la préposition du syntagme nominal, alors que l'ordre est plus libre avec la particule adverbiale. De plus, le " phrasal verb " peut être intransitif, c'est-à-dire n'être suivi d'aucun complément d'objet, alors que le "prepositional verb» devra avoir un complément qui correspondra au syntagme prépositionnel.

4 En ce qui concerne les " compound verbs ", la distinction est plus aisée : il s'agit d'un verbe précédé d'une particule, également d'origine directionnelle, mais qui est accolée au verbe, à gauche de celui-ci. Dans la présente étude il est question des première et troisième catégories.

D'un point de vue diachronique, M. Simon pose l'hypothèse que « les verbes préfixés en anglais contemporain, qui sembleraient moins 'productifs' que les phrasal verbs, tendent d'ailleurs à avoir une signification plus figée, ou davantage 'lexicalisée', par rapport à ces derniers » (p. 17). Le cadre théorique choisi pour l'étude est celui de la Théorie des Opérations Énonciatives fondée par Antoine Culioli. Plus précisément, M. Simon adopte une approche qu'il qualifie de "localiste», avec trois niveaux pour la particule, en fonction de sa compositionnalité :

- le niveau directionnel : la particule a son sens originel;

- le niveau aspectuo-modal, c'est-à-dire quand la particule n'a plus son sens directionnel mais a une valeur aspectuelle (par exemple quand elle permet de borner un procès) ou métaphorique telle que l'intensification ;

- le niveau notionnel : ni directionnel ni aspectuo-modal, il correspond au plus haut degré de lexicalisation. La méthodologie d'analyse utilise différents paramètres, définis pour les "phrasal verbs » et pour les «compound verbs", le but étant de mettre au jour les différentes opérations cognitives sous-jacentes dans le processus de création et de lexicalisation de ces verbes complexes. Les paramètres sont au nombre de trois :

- sémantique verbale, c'est-à-dire le type de procès, d'après la typologie des procès proposée par Vendler (1967) ;

- valence syntaxique, c'est à dire la possibilité pour un verbe d'être (ou non) transitif et/ou intransitif ;

- valence sémantique, c'est à dire le nombre et le type d'actants dans le procès. Ces trois critères sont utilisés conjointement, ce qui présente l'avantage et le mérite, même s'il rend la tâche ardue, de tenir compte à la fois du sémantisme du verbe et de la particule, ainsi que de l'aspect syntaxique et sémantique de l'ensemble. A chaque fois que c'est possible, l'analyse a également recours à la comparaison avec le verbe seul et/ou à la particule lorsqu'elle est utilisée différemment.

6 Une fois définie la méthodologie de l'étude, M. Simon peut alors se tourner vers son étude proprement dite, en commençant par les " phrasal verbs », dans le chapitre 2.

Le chapitre 2 est une étude très précise de ces verbes complexes suivant les critères définis dans le chapitre précédent. Il en résulte des tableaux descriptifs qui permettent à la fois de voir leur comportement et de les comparer aux verbes simples, pour en tirer des conclusions sur l'apport de chaque particule. 
7 La première sous-partie est consacrée à l'étude de la variation du sémantisme verbal. La question est: l'ajout d'une particule adverbiale permet-il (ou impose-t-il) au sémantisme verbal de changer? Pour ce faire, M. Simon regarde en détail les verbes à particules formés avec les couples de particules «traditionnels »: UP-DOWN, IN-OUT, ON-OFF, ainsi que quelques autres, OVER, ABOUT, ALONG, AWAY, BACK, BY, THROUGH. Il en conclut que les particules ont une influence non négligeable sur le sémantisme du verbe à particule, chacune ayant des comportements plus fréquents que d'autres. La deuxième sous-partie, sur l'aspect syntaxique, s'attache à l'opposition entre transitif et intransitif. Ici encore, l'étude montre une certaine corrélation entre particule, niveau fonctionnel du "phrasal verb» et comportement syntaxique de celui-ci. Dans une troisième sous-partie, M. Simon s'attache aux actants des procès décrits par les " phrasal verb », en lien avec les critères précédents.

8 Après cette partie sur la construction du sens dans les "phrasal verbs », le travail s'oriente vers une étude des mécanismes cognitifs à l'œuvre dans la construction même de l'unité. Ainsi, M. Simon met au jour l'existence de conversions syntaxiques comme, par exemple, la dérivation nominale (du substantif couple on arrive au " phrasal verb » couple up). Cette tendance s'accompagne d'autres opérations comme la localisation par la particule (externe comme interne, p. 77). Sur les «phrasal verbs" pour lesquels le verbe est également un verbe seul, il existe parfois des phénomènes de double prédication. Dans cette optique, $\mathrm{M}$. Simon reprend et développe l'hypothèse de Lipka (1972), que le " phrasal verb » porte la trace de deux prédications sous-jacentes : il sert à mettre en relations deux lexis, tout en n'en gardant en surface que la trace, puisqu'il n'y a plus de "visible " qu'un verbe et une particule. Encore une fois, les critères précédemment décrits et utilisés se montrent utiles pour étayer sa thèse, en particulier le sémantisme verbal construit: la particule localise le procès porté par le verbe, mais aussi permet de reconstruire la deuxième prédication, dont elle est la trace. Ainsi, dans l'exemple " did you check who signed the box in?", on a deux lexis (" who signed X" et « the box BE in ») qui sont mises en relation, et qui participent de la construction du sens au niveau énonciatif.

9 Le chapitre 3 reprend la même trame que le précédent, mais s'attelle aux « compound verbs » ou verbes préverbés. Après un retour sur la définition et l'évolution des verbes préverbés en anglais, M. Simon reprend les critères qu'il utilise dans son étude sémantico-syntaxique. Pour des raisons de fréquence d'emploi, les dérivés de « compound verbs » (nominaux, participiaux) sont intégrés à l'étude, ce qui l'enrichit tout en la complexifiant. Les critères sont similaires aux critères d'étude des "phrasal verb»: sémantique du verbe construit, valence sémantique, valence syntaxique. Le niveau fonctionnel de la particule apporte également un éclairage précieux sur le fonctionnement du verbe préverbé. Comme dans le chapitre précédent, on se rend compte que les phénomènes qui entrent en jeu sont non seulement complexes et parfois difficiles à cerner, mais aussi qu'ils ont une importance fluctuante selon les cas. M. Simon conclut donc à des corrélations plutôt que des règles strictes quant à l'influence d'une partie sur l'autre, ou d'un critère sur les autres.

10 Après une comparaison avec les "phrasal verbs", il conclut également que les deux types de verbes complexes se comportent différemment, notamment sur deux points. La valence syntaxique, tout d'abord, fluctue bien plus dans le cas des verbes à particules que celui des préverbés, ce qui est probablement le signe d'une plus grande souplesse mais peut-être aussi d'un moins grand degré de lexicalisation chez les verbes à 
particules. Cette tendance est confirmée par la plus grande diversité des champs actanciels (p.176) qui est notée pour les préverbés. Ensuite, la fonction de trois particules est particulière dans les préverbés : under, out et over. Ces trois particules ont en effet un rôle de comparaison dans ces constructions (dans des verbes comme outpace ou over/underestimate), c'est-à-dire qu'il y a "une opération 'modale' deprédication comparative » (p. 177).

11 Tout au long de cet ouvrage, $\mathrm{M}$. Simon a recours à des analyses très rigoureuses et fines $\mathrm{du}$ sens des formes étudiées, et des mécanismes mis en jeu pour produire ce sens. Les conclusions auxquelles il arrive en sont renforcées, et tout à fait convaincantes. Quelques problèmes demeurent, mais sont plus de l'ordre de la forme que des résultats. Les statistiques demanderaient à être plus clairement justifiées : elles reposent sans doute sur l'étude systématique du corpus utilisé, mais celui-ci n'est décrit que dans le chapitre 3. On attendrait la même description du corpus utilisé pour les verbes à particules. De même, certains exemples choisis permettraient parfois des interprétations quelque peu différentes, mais c'est le lot incontournable de tout linguiste qui travaille sur de la langue réelle plutôt que sur des exemples fabriqués. Enfin, sur les critères utilisés, on peut regretter que l'aspect sémantique ne concerne que la typologie des procès de Vendler, ce qui laisse de côté tout une partie du sens des verbes (Consigny 2000). Cependant, l'ajout de critères supplémentaires rendrait la tâche quasi impossible, en multipliant encore les combinaisons de critères, et par conséquent en augmentant d'autant la taille de l'ouvrage qui est déjà très dense en l'état. Tout au plus on peut en conclure qu'il reste des perspectives d'ouverture sur cette excellente étude.

\section{BIBLIOGRAPHIE}

CONSIGNY Antoine, "Looking at Phrasal Verbs in a Data-Driven Perspective: A Case Study of Take Up”, Revue Française de Linguistique Appliquée, vol. V-2, 2000 : 7-18.

LIPKA Leonhard, Semantic Structure and Word-Formation: Verb-Particle Constructions in Contemporary English, Münich, Wilhelm Fink Verlag, 1972.

VENDLER ZENO, Linguistics and Philosophy, Cornell University Press, 1967

\section{AUTEURS}

\section{ANTOINE CONSIGNY}

Antoine Consigny est maître de conférences au département d'Etudes Anglaises et Nord-

Américaines de l'Université de Strasbourg, où il enseigne la linguistique anglaise de la 1ère année à l'agrégation. Il a soutenu à Liverpool en 2002 une thèse sur la sémantique des verbes à particules, et a poursuivi ses recherches en sémantique, en particulier en sémantique verbale, et en grammaire. 